\title{
Alterstice
}

Revue internationale de la recherche interculturelle

International Journal of Intercultural Research

Revista International de la Investigacion Intercultural

\section{Trajectoires migratoires et options identitaires : paroles d'aînés d'origine libanaise}

\section{Habib El-Hage}

Volume 6, numéro 2, 2016

URI : https://id.erudit.org/iderudit/1040630ar

DOI : https://doi.org/10.7202/1040630ar

Aller au sommaire du numéro

Éditeur(s)

Alterstice

ISSN

1923-919X (numérique)

Découvrir la revue

Citer cet article

El-Hage, H. (2016). Trajectoires migratoires et options identitaires : paroles d'aînés d'origine libanaise. Alterstice, 6(2), 29-40.

https://doi.org/10.7202/1040630ar

\section{Résumé de l'article}

Les recherches sociologiques sur les aînés dans un contexte migratoire sont peu nombreuses. Faisant suite à notre recherche doctorale, cet article étudie les options identitaires des aînés d'origine libanaise vivant à Montréal, au Québec. Notre hypothèse de départ propose que la reconstruction identitaire chez les aînés d'origine libanaise en contexte migratoire et le choix des options identitaires se font à partir de l'expérience vécue dans le pays d'origine et de l'expérience vécue dans la société d'accueil. Nos résultats témoignent de la complexité de la composition et de la recomposition identitaire des aînés d'origine libanaise, du fait que les aînés choisissent diverses options identitaires influencées par leur nouvel environnement social et qu'un certain détachement identitaire de la société d'origine se dessine, dû à des conjonctures historiques, politiques et sociales. 
ARTICLE HORS THÈME

\section{Trajectoires migratoires et options identitaires : paroles d'aînés d'origine libanaise}

Habib El-Hage ${ }^{1,2,3}$

\section{Résumé}

Les recherches sociologiques sur les aînés dans un contexte migratoire sont peu nombreuses. Faisant suite à notre recherche doctorale, cet article étudie les options identitaires des aînés d'origine libanaise vivant à Montréal, au Québec. Notre hypothèse de départ propose que la reconstruction identitaire chez les aînés d'origine libanaise en contexte migratoire et le choix des options identitaires se font à partir de l'expérience vécue dans le pays d'origine et de l'expérience vécue dans la société d'accueil. Nos résultats témoignent de la complexité de la composition et de la recomposition identitaire des aînés d'origine libanaise, du fait que les aînés choisissent diverses options identitaires influencées par leur nouvel environnement social et qu'un certain détachement identitaire de la société d'origine se dessine, dû à des conjonctures historiques, politiques et sociales.

\section{Rattachement de l'auteur}

${ }^{1}$ Équipe METISS (Migration et ethnicité dans les interventions en santé et en services sociaux), SHERPA et CRIEC, Montréal, Québec

2 Université de Sherbrooke, Canada

${ }^{3}$ Collège de Rosemont, Montréal, Canada

\section{Correspondance}

elhageh@hotmail.com

\section{Mots clés}

migration; comparaison sociale; identités.

\section{Pour citer cet article}

El-Hage, H. (2016). Trajectoires migratoires et options identitaires : paroles de personnes âgées d'origine libanaise. Alterstice, 6(2), 29-40. 


\section{Introduction}

Les politiques d'immigration nous annoncent que la pluriethnicité et l'apport croissant de la population venant de l'étranger demeureront une caractéristique de plus en plus marquante de notre société. Dans le contexte québécois où, entre autres, la question nationale est porteuse d'interrogations et de conflits, les problématiques de l'identité sont plus que jamais présentes dans l'espace public et figurent parmi les préoccupations des citoyens. La littérature scientifique présente le vieillissement à partir de considérations gérontologiques : selon les observations de Charpentier et Quéniart (2011), « les sociétés vieillissent et, au Québec, ce phénomène est un des plus rapides au monde. Les personnes de 65 ans et plus constituent actuellement $14 \%$ de la population » (p. 15). Les aînés du Québec issus de l'immigration représentent quant à eux près de $17 \%$ des aînés (Gouvernement du Québec, 2015, p. 16).

La problématique de l'identité peut être abordée sous différentes perspectives (catégories sociales et statuts, rôles ou interactions entre différents interlocuteurs). Dans cet article, l'identité sera considérée comme étant " une manière d'interagir, d'appartenir ou de ressentir une appartenance à un groupe social, à une idéologie, à une manière de vivre ou de penser " (Berger et Luckmann, 1986). Nous ajoutons à cela que l'identité évolue dans le dialogue, la négociation et la confrontation entre le semblable et le différent. Le processus identitaire est interactif, relationnel, dynamique et implique de prendre en considération l'Autre dans sa différence et son environnement. Plusieurs études ont abordé l'identité en contexte migratoire. Labelle, Salée et Frenette (2000) affirment entre autres que "les diverses formes de l'identité renvoient à un processus de construction sociale qui transforme constamment ses contours » (p. 146). Portes et Zhou (1993), Martiniello (1995) et Berry (2000) accordent pour leur part une importance aux politiques publiques dans la construction identitaire, tandis que Waters (1996) et Manço (1999) octroient une place prépondérante à la structure familiale parmi les facteurs qui influencent le choix des options identitaires.

L'hypothèse sur laquelle nous nous sommes basés pour cette recherche est que les options identitaires en société d'accueil sont tributaires des appartenances identitaires dans la société d'origine et des expériences vécues dans la société d'accueil. Le malaise identitaire figure parmi les préoccupations des citoyens. Plus globalement, des recherches et débats sur les identités des immigrants, surtout les jeunes de la première ou de la deuxième génération, ont fait couler beaucoup d'encre. Depuis longtemps, les chercheurs s'intéressent à cette question afin de savoir comment les identités des immigrants se transforment au contact des sociétés d'accueil et comment la société d'accueil se trouve, elle aussi, en mutation. $Y$ a-t-il des modèles d'identifications particuliers chez les immigrants? Y a-t-il des facteurs dominants qui influencent le choix des options identitaires, par exemple celles des aînés d'origine libanaise vivant à Montréal?

\section{Considérations théoriques}

Plusieurs auteurs nous ont inspiré pour élaborer notre cadre conceptuel et mener notre analyse. Nous retenons ainsi le concept de l'assimilation segmentée développé par Portes et Zhou (1993), qui affirment qu'il est « essentiel de considérer différents niveaux d'analyse influençant les modes d'incorporation » (p. 86). Ils en distinguent trois : "la mise sur pied de certains programmes gouvernementaux, l'opinion publique envers les minorités visibles et les ressources morales et matérielles offertes par la communauté ethnique ».

Nos analyses se sont aussi appuyées sur l'apport théorique de Waldinger (1996) et celui de Lévesque et White (1999) sur les réseaux sociaux, sur la force des liens établis, sur leurs contraintes et leurs opportunités. Notamment, le réseau ethnique, structure sociale importante, souvent spatialement concentré, peut être un atout ou un handicap selon s'il offre ou non à ses membres les ressources nécessaires de travail, d'information, de support facilitant l'accès à la mobilité sociale, avec bien entendu le risque de cloisonnement.

D'autres théories ont été importantes dans le cadre de notre recherche. Notre analyse s'est enrichie grâce aux recherches de Camilleri sur l'identité ainsi qu'aux travaux de Cerclé et Sonat sur la théorie de la comparaison sociale. En effet, Camilleri présente l'identité en termes de stratégies identitaires, de construction identitaire, c'està-dire "l'idée que les individus et les groupes ont une certaine capacité d'action sur les choix de leur groupe 
d'appartenance et de référence " (Camilleri et al., 1990, p. 23). La relation d'enchevêtrement entre la culture et l'identité est le point de départ adopté, le thème central de son approche théorique (Camilleri et al., 1990). Cerclé et Sonat (2002) démontrent, à ce titre, que le processus de comparaison sociale s'applique à la formation d'opinion, à l'appréciation et à l'évaluation des aptitudes personnelles de l'individu. Cette théorie insiste sur le besoin que nous ressentons d'utiliser les autres comme source de comparaison afin d'évaluer nos propres attitudes et capacités.

\section{Méthodologie}

En cohérence avec l'approche interactionniste et compte tenu de la nature exploratoire qui, selon Gauthier (1996), permet une description en profondeur, nous avons choisi une démarche qualitative pour notre étude afin de "privilégier le point de vue des acteurs sociaux dans l'appréhension des réalités sociales » (p. 18). Nous pensons que les aînés sont les mieux placés relativement à l'explication et à la description de leur vécu et de leur perception. Deux groupes de personnes âgées d'origine libanaise ont été rejoints. Le premier est composé d'individus arrivés à un jeune âge, durant les années 1950 à 1970, ce qui correspond à la deuxième "vague» ${ }^{1}$ de Libanais arrivés après la Seconde Guerre mondiale et suite à l'abolition des lois discriminatoires ${ }^{2}$, et l'autre groupe est constitué d'individus arrivés au Québec à un âge plus avancé, durant la troisième période migratoire des Libanais vers le Québec. Pour ces deux cohortes, il s'agit de la première génération de Libanais vivant à Montréal. Deux dimensions nous interpellaient, celle portant sur l'expérience vécue dans la société d'origine et celle portant sur le vécu et le perçu au sein de la société d'accueil.

Le corpus d'analyse de cette recherche est constitué du récit de 37 personnes âgées, 18 femmes et 19 hommes. L'âge des femmes oscille entre 65 ans et 74 ans et celui des hommes entre 65 ans et 82 ans. Les répondants sont d'origine libanaise de Montréal mais proviennent de différentes périodes d'immigration, de différentes allégeances confessionnelles ${ }^{3}$, de différentes classes sociales. Les répondants parlent majoritairement l'arabe au quotidien, du fait qu'ils sont de la première génération et sont tous nés au Liban. Presque tous les répondants ont des enfants qui vivent à Montréal ou ailleurs, soit dans le pays d'origine soit dans une autre province du Canada. Les hommes sont plus scolarisés que les femmes. Les hommes et les femmes ont occupé des emplois diversifiés au Liban et au Québec. Toutes les personnes rencontrées vivaient d'une manière autonome, en appartement ou en bâtiment intergénérationnel au moment de l'entrevue. Nous avons effectué des entrevues en profondeur de type semi-dirigé en langue arabe (dialecte et littéraire). Saisir la parole dans la langue d'origine a été pour nous incontournable et très heuristique, nous donnant accès à un univers de pratiques et de sens (comprenant une attention particulière aux expressions, métaphores et proverbes) à la fois très singulier et socialement situé.

\section{Expérience de la société d'origine}

Deux éléments principaux se sont dégagés de nos analyses : la construction identitaire dans le pays d'origine et le projet migratoire. À partir de la narration des personnes répondantes sur leurs vies dans le pays d'origine, on retrouve pêle-mêle des paramètres liés à la religion, à la classe sociale, à l'influence nationaliste et à la localité. Pour la plupart, nées avant la constitution politique et juridique du Liban, elles se définissent comme étant libanaises par idéologie ou par opposition à un autre groupe. Dans d'autres cas, l'identité parentale (mère ou père) est priorisée. L'identification se fait aussi à une classe sociale, bourgeoise ou populaire. En d'autres mots, leurs

\footnotetext{
${ }^{1}$ II y a eu trois périodes migratoires des Libanais vers le Canada et le Québec. La première remonte au $19^{\mathrm{e}}$ siècle, après les troubles communautaires et confessionnels au Mont-Liban (1840-1860), la deuxième s'étend d'après la Seconde Guerre mondiale à la fin des années 1960 et la troisième correspond à la guerre civile dans laquelle le Liban a plongé (1975-1990).

${ }^{2}$ En 1946, le gouvernement fédéral créa une loi intitulée la Loi sur la citoyenneté canadienne, qui entra en vigueur le $1^{\mathrm{er}}$ janvier 1947. Après la création de cette loi, le Canada continua toutefois à offrir un traitement préférentiel aux résidents blancs européens ou américains qui voulaient immigrer en ce pays. Cela était évident dans la Loi sur l'immigration de 1952 : elle exerçait une discrimination envers les Asiatiques et ceux qui venaient du continent asiatique n'ayant aucun parent proche vivant au Canada ou avec les autres groupes ethniques désignés par le gouvernement.

${ }^{3}$ Au Liban, la dimension religieuse occupe un espace privilégié dans les rapports public-privé. Il y a 18 confessions religieuses officielles, avec leurs propres tribunaux religieux.
} 
options identitaires sont influencées par des trajectoires singulières qui les ont façonnées. Elles sont touchées par certains déterminants : les événements géopolitiques comme l'indépendance du Liban, la présence d'étrangers ${ }^{4}$ ou encore par leurs conditions ou positions de classe et par leur genre. Il est ainsi possible d'affirmer, sans crainte d’observer des cristallisations, qu'une pluralité d'identités de sujets est démontrée.

On a remarqué pour certaines personnes répondantes, à défaut d'un fort symbole d'identification, que la localité et l'appartenance territoriale représentent un marqueur identitaire important. À titre d'exemple, le témoignage de cet homme :

II faut dire que je suis né dans les années 20, en 1922. À cette époque, il n'y avait pas de frontières avec la Palestine. On allait là-bas, on travaillait, on était des villageois. On nous appelait comme ça : Al karyaoui (le villageois). On n'avait pas cette notion de Libanais ou de musulman ou de chrétien. II y avait les Juifs, on se méfiait, mais on travaillait ensemble. II y avait encore des Français, il y avait des Africains, il y avait de toutes les nationalités sur nos terres. On était très pauvres, mais nous étions tous pauvres (Homme, chiite, 85 ans, né au Liban-Sud, à Montréal depuis 1992)

Dans l'un des rares ouvrages à avoir analysé à fond les structures de la société libanaise, Abou démontre que l'origine commune, essentiellement arabe, de la population au Liban révèle un modèle individuel de comportement très commun, dans ce sens :

Dès que l'on touche à l'individu, on découvre qu'il définit son comportement à l'intérieur d'une réalité essentielle : le groupe familial, à partir duquel se dessine tout son réseau de relations. Parmi les populations de culture arabe, faire remonter l'origine de sa famille à une tribu bédouine est le moyen d'en affirmer l'ancienneté et la noblesse; des familles notables druzes, maronites, ainsi que de toutes les communautés apprirent à l'utiliser. (2002, p. 95)

Le caractère affirmé de l'identité est perceptible dans les propos d'autres qui nous indiquent que leur identité libanaise est un élément indivisible. Une identité qui est présente avant même l'indépendance du pays. Les propos de ce syndicaliste qui militait pour la création d'un Liban indépendant nous apportent un éclairage différent du témoignage précédent :

J'étais fier d'être un Libanais, je me suis réveillé sur l'indépendance en 1943... Elle ne part pas cette identité, celui qui dit qu'elle va disparaître... ce n'est pas vrai. Elle était présente avant même l'indépendance. (Homme, 83 ans, témoin de Jéhovah, né à Liban-Nord, à Montréal depuis 1996)

\section{La migration : un projet de cohésion familiale}

La revue de la littérature (Abou, 2002; Joseph, 1998; Le Gall, 2001) nous apprend l'importance de la structure familiale et la cohésion des liens familiaux. Nous l'avons observé dans le discours des personnes interrogées. Les enfants choisissent de partir à la recherche d'un avenir meilleur. Plusieurs personnes interrogées affirment avoir quitté le pays d'origine afin de suivre les enfants installés ailleurs.

Ce qui m'a poussé à quitter c'est le départ de mes enfants, moi je voulais rester, par contre, mes enfants en avaient assez. Et lorsqu'ils ont quitté, moi je n'étais plus capable de rester. Je suis partie après. (Femme, catholique romaine, 71 ans, née à Beyrouth, à Montréal depuis 1982)

La migration ou l'idée de quitter le pays en déchéance dû à une guerre civile se trouve au cœur d'une stratégie de survie. Retrouver un sens à sa vie devient le moteur de création de nouveaux liens, la préparation à un départ. Le lien entre les membres de la famille est évoqué à maintes reprises dans le discours des répondants.

J'habite à côté de ma fille à deux rues d'ici, mais on est toujours chez elle, ma femme s'occupe beaucoup de ses enfants. (Homme, maronite, 65 ans, née au Liban Sud, à Montréal depuis 1989)

\footnotetext{
${ }^{4}$ Par étrangers, nous désignons les populations qui ont fui les pays avoisinants durant les révolutions ou les guerres. Ce fut le cas des palestiniens, des arméniens, des kurdes, des syriaques, etc.
} 


\section{Le trauma de la guerre}

Une unanime dénonciation s'élève contre le désastre de la guerre qui a frappé le Liban pendant 15 ans. Les personnes répondantes qui sont arrivées pendant la guerre 1975-1990, ou après celle-ci, témoignent d'une expérience douloureuse sinon traumatique. Encore une fois, le Liban, pays d'un projet de cohabitation et du vivre ensemble, a été mis à l'épreuve d'une façon radicale. Certaines parlent d'une malédiction s'étant abattue sur les Libanais.

C'était très difficile ... on ne savait pas s'il fallait vendre nos biens ou non. À qui, à combien, personne n'achetait au Liban, c'était nul. On était dans la confusion totale. Il fallait quitter tout et tout le monde... pour moi c'était la folie. (Femme, maronite, 74 ans, née à Beyrouth, à Montréal depuis 1988)

La guerre n'a pas été continue, mais entrecoupée par de nombreuses périodes d'accalmie, de trêves ou de cessezle-feu, toujours suivie par une reprise d'hostilités plus violentes. À chacune des périodes d'affrontement correspondent des fuites massives de citoyens vers l'étranger. Pour la seule période des affrontements entre groupes chrétiens rivaux en 1990, l'une des phases les plus meurtrières du conflit, les départs se sont chiffrés à 220000 personnes (Pinta, 1995).

Aux bombardements s'ajoutent l'oppression politique, la terreur, le poids de la rumeur. Pour toutes et tous, enlèvements, tortures, attentats et voitures piégées marquent le quotidien. Près d'un million de personnes ont été déplacées à l'intérieur même des frontières. Cette population a dû trouver refuge chez des membres de la famille particulièrement, dans la précarité après avoir perdu ses biens :

Plus que la guerre, c'était surtout fuir les villages, se retrouver réfugié ailleurs dans ton pays et dans l'expropriation d'autres maisons. On a tout perdu c'est difficile à croire parfois. Mais c'est sûr que c'est à cause de la guerre tout ça. Personne ne savait que ça allait durer 16 ans. Chaque année on disait que c'est la dernière et comme ça pendant 16 ans. (Homme, maronite, 72 ans, né à Beyrouth, à Montréal depuis 1988)

Le projet d'émigration représente une décision lourde d'enjeux, troublante affectivement, ardue dans sa trajectoire. Le désir d'avoir pour soi et ses enfants enfin une vie sécuritaire est capital et décisif. Une souffrance individuelle et collective très grande émerge donc de cette expérience de la guerre.

\section{Projet migratoire}

Dans un premier temps, il apparaît que nous sommes en présence d'une émigration volontaire, c'est-à-dire la filière de l'immigration et non celle du refuge, quoique pour la plupart, la guerre en tant que phénomène social ne laisse pas toujours le choix. Le départ est présenté comme action réfléchie et planifiée. Les individus choisissent d'émigrer en fonction de leur réalité : besoins individuels tout autant que raisons familiales, comme protéger la famille ou offrir une éducation stable aux enfants. Ce processus décisionnel complexe demande aux individus de faire des compromis (vendre des biens, choisir une destination, se séparer de la famille élargie, des amis) afin de sortir de la folie guerrière, de retrouver un sens à sa vie, d'améliorer la situation de l'ensemble de ses proches.

C'était la guerre, la guerre. Les enfants étaient au Liban, on est venu en équipe : la première équipe est arrivée en premier, moi et mon fils, et ensuite mon mari et les filles. (Femme, sunnite, 69 ans, née à Beyrouth, à Montréal depuis 1990)

Dans la partie suivante, nous allons voir comment a été vécue l’expérience de la société québécoise.

\section{L'expérience de la société d'accueil}

D'après les expériences d'interaction en pays d'accueil, les personnes rencontrées semblent avoir développé des relations satisfaisantes. L'expérience de vie au Québec est pleine d'éléments positifs. La notion de reconnaissance et de respect est au centre du vécu des personnes âgées d’origine libanaise qui se sont installées au Québec. 
Je le dis depuis longtemps, le meilleur système est ici. Il faut apprécier la facilité des choses, mais il faut dire aussi que les gens ont travaillé fort pour arriver à quelque chose comme ça, accessible, facile, tout le monde est dedans. (Femme, confession sunnite, 68 ans, née à Beyrouth, à Montréal depuis 1988)

Les options identitaires reformulées au contact avec les institutions publiques, les rapports égalitaires, la présence de la protection sociale, l'organisation des services dans la société, les lieux de culte, l'accueil et le respect des individus aident à l'acceptation, en retour, de la société d'accueil.

Honnêtement, si on avait des institutions au Liban comme celle qui est ici, je pense que je resterais au Liban. On sent qu'il y a un respect et une sincérité du service ici. Ça me plaît. (Homme, chiite, 71 ans, né à Beyrouth, à Montréal depuis 1994)

Les individus qui sont arrivés durant les années 1950 et 1960 ont su bénéficier d'un contexte économique de pleine croissance au Canada et au Québec, tandis que ceux arrivés dans les années 1980 ont été confrontés aux difficultés, entre autres, d'emploi, d'interaction avec le système de la société d'accueil et même d'interaction avec des structures de la communauté libanaise.

\section{L'institution publique, garante du vivre ensemble}

La compréhension des institutions et du système politique, social, culturel est différente d'une personne à une autre. II faut dire que le facteur temps est significatif dans la compréhension du fonctionnement de celles-ci. Aussi l'âge à l'arrivée peut-il jouer un rôle important dans l'incorporation au sein de la société d'accueil.

J'ai fini mes études ici en 1961-1962, je me suis occupé du club international de la Ville de Québec. J'ai réussi mon intégration quand je me suis occupé de l'intégration des autres. (Homme, maronite, 73 ans, né au Liban-Nord, à Montréal depuis 1958)

Le fait que le Québec soit une société francophone a certainement influencé le choix d'établissement de certaines personnes répondantes. Plusieurs parmi elles, arrivées avec un statut d'étudiant étranger, se sont davantage identifiées au milieu francophone et ont développé plus facilement une compréhension du fonctionnement de la société et de ses institutions. La connaissance de la langue représente en effet une porte d'entrée directe sur la communauté d'accueil et semblerait influencer la qualité et la quantité des premiers contacts à l'extérieur du foyer.

Je suis venu à Montréal, j'ai enseigné le français au collège. Très vite, on m'a permis d'introduire un cours de sociologie. II n'y avait qu'un seul collège, c'était le collège des jésuites qui enseignant cela. (Homme, 73 ans, maronite, née au LibanNord, à Montréal depuis 1958)

\section{Réalisation de soi}

Plusieurs femmes interrogées relatent l'expérience enrichissante que vivent les femmes au Québec. Plus de ressources, plus de respect, la valorisation de l'égalité entre les hommes et les femmes, plus d'autonomie, pour certaines, une véritable stimulation à la subjectivation, donc à plus de liberté.

Je dis à n'importe quelle femme, si elle veut être respectée, la meilleure vie est ici au Canada... La personne aînée vit avec respect ici, elle peut aider ses enfants même. Même ma belle-fille ne sera pas dérangée. Je ne demanderai jamais à mes enfants un salaire ou bien une aide quelconque, parce que je ne veux pas que mes enfants souffrent comme moi j'ai souffert. (Femme, maronite, 65 ans, née au Liban-Sud, à Montréal depuis 1989)

L'attitude réceptive ressentie par les personnes répondantes démontre qu'il y a un sentiment d'acceptation par la société d'accueil. Ces attitudes s'incarnent par le respect, l'humanisme et la confiance.

Je suis d'accord avec cette vision de société qui se construit de cette façon et qui tend à être humaine le plus possible. Il y a de la place pour tout le monde sans arrogance ni discrimination. (Femme, catholique romaine, 66 ans, née à Beyrouth, à Montréal depuis 1967) 
Quant au travail, les divers récits des aînés le présentent comme étant une clé à l'insertion dans la société d'accueil. Celles qui ont fait l'expérience du travail ont qualifié l'insertion socio-économique de cruciale personnellement, pour leurs enfants et surtout pour les femmes. Notamment, le système québécois ouvre aux femmes tout un éventail de possibilités reliées au travail et aux nouvelles réalités socioculturelles. L'absence de la famille élargie ainsi que la faiblesse des structures sociales de la communauté rendent nécessaire la création de nouvelles organisations en fonction de la réalité immigrante. Les propos de cette femme sont éloquents :

La vie des Libanais est très active, mais ici la vie est plus facile et plus rapide, il n'y a pas ici cette notion de honte comme au Liban. Comme femme au Liban, c'est difficile pour moi de travailler, mais ici ce n'est pas grave. (Femme, chrétienne orthodoxe, 68 ans, née au Liban-Nord, à Montréal depuis 1990)

Loin du pays d'origine, les femmes interviewées sont devenues beaucoup plus autonomes. Sortant de leurs rôles, elles se sont initiées à une participation sociale plus active. Par la force des choses, elles ont pris des décisions seules. Ainsi, elles ont exploré l'espace public malgré les distances à parcourir en transport en commun et aussi malgré les difficultés de communication. La multiplication des contacts avec l'extérieur fait qu'elles ont développé assez rapidement leur débrouillardise.

\section{Expériences de discrimination}

D'après les expériences d'interaction que les personnes répondantes ont eues avec le nouvel environnement culturel et social, elles définissent les liens sociaux en termes harmonieux. Leur expérience d'interaction révèle la manière dont elles interprètent la réalité au Québec. Presque toutes les personnes répondantes arrivées dans les années 1950, 1960 et 1970 soulignent l'existence du racisme et de la discrimination, mais affirment ne jamais avoir vécu ces expériences d'une façon personnelle. Toutefois, un répondant fait référence à une expérience malencontreuse lors d'un voyage à l'étranger :

Pour moi, la forme la plus sournoise du racisme vécue lorsque c'est quelqu'un m’a dit à l'aéroport "d'où vous venez?", alors j'ai dit “je suis Québécois" et là il a répondu “oui oui je sais, mais où êtes-vous né, d'où vous venez?" alors, je lui ai dit "du Liban". (Homme, maronite, 81 ans, né au Liban-Nord, à Montréal depuis 1958)

Il faut noter que plusieurs répondants, arrivés au Québec à la fin de la guerre civile du Liban (1975-1990), n’ont pas vécu l'expérience de recherche d'emploi ou de recherche de logement. Ceci explique en grande partie la perception d'une société idéaliste. Les témoignages de ces répondants démontrent ces perceptions :

Personnellement, je n’ai pas vu ici du racisme. (Homme, maronite, 72 ans, né au Liban, à Montréal depuis 1999)

Les récits des personnes répondantes montrent la présence d'une expérience d'ouverture à la société d'accueil. La faiblesse d'événements de discrimination et de racisme joue un rôle important dans cette reconfiguration positive de l’identité. La structure communautaire libanaise joue-t-elle un rôle?

\section{Structure communautaire libanaise}

La présence d'une structure communautaire libanaise, ou plutôt d'associations religieuses, sociocommunautaires et médiatiques, remonte au $19^{\mathrm{e}}$ siècle au Québec. Celles-ci sont concentrées particulièrement à Montréal, et dépendent du nombre de membres de la communauté libanaise et de la distribution géographique résidentielle sur le territoire de l'île de Montréal. Elles exercent une influence sur les membres de la communauté en rapport avec leurs missions, comme l'intégration, l'information, le rassemblement, l'animation collective, etc. II faut dire que la communauté libanaise était plus homogène au début de son installation en terre d'accueil sur les plans de la religion, de la classe sociale et de l'identité nationale.

Par la suite, l'arrivée d'autres groupes d'immigrants libanais de diverses confessions religieuses et de différentes classes sociales a eu des répercussions au sein même de la communauté, se manifestant par différentes tensions et oppositions. Les valeurs, les principes et les croyances de chaque groupe entrent en compétition. Les divisions 
créées viennent nous rappeler celles du pays d'origine. Les propos de ce répondant reflètent ce que plusieurs personnes que nous avons interrogées pensent :

J'ai l'impression que plusieurs veulent reproduire et reproduisent le même clivage que là-bas et en ça moi je n'ai pas envie d'entrer, un étudiant au doctorat qui travaille sur le Liban m'apprend que toutes les factions politiques du Liban sont représentées ici. (Homme, maronite, 73 ans, né au Liban-Nord, à Montréal depuis 1958)

Les personnes interviewées n'hésitent pas à dénoncer ce phénomène dans la communauté.

\section{Les options identitaires postmigratoires}

Le sentiment d'appartenance et la transformation identitaire des répondants sont définis différemment en fonction des interactions avec la société d'accueil. Les personnes qui sont arrivées au Québec depuis longtemps ont affirmé d'abord avoir participé à la société québécoise, donc disent éprouver une appartenance ouverte et sans ambigüité. En examinant leurs témoignages, nous pouvons identifier plusieurs causes. Leur réseau de contacts, leur ascension professionnelle, l'identification à la classe moyenne québécoise, leur maîtrise de la langue a facilité l'acquisition des codes culturels et des protocoles d'interaction avec la population d'accueil. Leur jeune âge lors de leur arrivée au Québec a facilité leur participation. De même pour ceux et celles qui se réfèrent à leur appartenance au Canada. Même lorsque des répondants évoquent les limites de leur contact avec la société d'accueil, ces limites n’ont pas empêché une identification à la société d'accueil. L'accueil, le confort et la reconnaissance sont soulignés.

Je vis très bien avec ma québécitude, je la valorise et je l'affiche. (Femme, chrétienne orthodoxe, 76 ans, née à Beyrouth, à Montréal depuis 1984)

Je te donne un exemple, si tu arrives devant quelque chose de bien tu ne peux que soupirer du confort. Je les aime les Canadiens parce qu'ils me font vivre, c'est honteux de nier ce qu'ils me font, je me sens canadien. (Homme, témoin de Jéhovah, 83 ans, né à Beyrouth, à Montréal depuis 1996)

Par ailleurs, les entrevues ont révélé une certaine opposition de la part de quelques répondants à la culture québécoise. Deux zones semblent être fragiles et remises en question, soit les rapports de genre et la perception du voisinage et de l'entraide.

Toutes les personnes arrivées pendant ou après la guerre du Liban (1975-1990) ont conservé un lien familial avec les membres de leurs familles qui sont dans le pays d'origine. Le contact est maintenu grâce aux télécommunications ou bien par des visites.

Les aînés sont unanimes quant à la fonction du séjour et des voyages. Le retour au Liban semble être une réalité difficile à réaliser, puisque le mode de vie leur apparait peu enviable. Un répondant ne semble pas être encouragé à retourner au Liban, sauf pour une visite avec les enfants qui n'ont pas connu le pays d'origine de leurs parents et la culture libanaise dans son ensemble, à la source :

Non, je vais un peu chaque année, mais je ne vais pas retourner pour toujours. Je n'irai pas tout seul. Mon fils aussi, il me dit la même chose, si je veux aller au Liban il faut que ce soit avec mes enfants. (Homme, sunnite, 81 ans, né à Beyrouth, à Montréal depuis 1991)

Dans la dynamique migratoire des personnes rencontrées, le niveau de vie et le contexte montréalais libanais influencent la redéfinition du contenu initial du projet migratoire. Après quelques années de vie à Montréal, les zones grises se sont clarifiées et une décision d'installation définitive prend forme. Les avantages sociaux et la relative sécurité économique encouragent une certaine mobilité sociale. Les personnes répondantes ont vu leurs enfants profiter des avantages que le système offre à l'ensemble des citoyens (éducation gratuite, aide sociale pour les démunis, hospitalisation gratuite, etc.). Les aînés disent que même en faisant face à des ennuis d'ordres divers comme le déplacement, la bureaucratie, des barrières linguistiques à Montréal, le niveau de vie y est supérieur et leur permet de réaliser des actions concrètes, pour elles et pour leurs enfants. Le projet de retour s'efface avec le temps et les liens avec le pays d'origine s'amenuisent. Ainsi, la refonte des identités prend une forme, la 
multiplicité des identités se reformule. Les personnes rencontrées parlent d'une identité politique canadienne libanaise, québécoise libanaise, d'une identité sociale d'aînée et toujours d'un ancrage familial profond.

\section{Synthèse analytique}

La contribution particulière de notre recherche réside dans le fait que nous avons tenté de mettre en œuvre une approche originale, associant, à partir de la parole de l'individu, ses rapports dynamiques avec la société d'origine et la société d'accueil.

Nous présentons quatre thèmes majeurs qui se sont dégagés du processus de construction de stratégies identitaires des personnes âgées d'origine libanaise à Montréal.

\section{La société d'accueil : une adhésion personnelle et réfléchie}

Les personnes interrogées invoquent la constitution d'un appareil d'État fort, offrant la liberté, la sécurité et une conception humaniste de l'organisation du vivre ensemble. Le rôle des institutions, programmes et services publics ne cesse d'être vanté. Ce qui est mis en exergue se rapporte au rôle des aînés, qu'elles estiment reconnu et valorisé, à la sécurité de la vieillesse et à un traitement de la quotidienneté en général respectueux. Il y a un choix, une capacité d'agir, une action des sujets.

Le rapport est positif parce qu'il y a une attention particulière pour les personnes âgées, parce qu'elles se sentent accueillies par une société démocratique reconnaissant leurs droits. Ce point est à mettre en lien avec la position des auteurs Portes et Zhou (1993) sur les politiques et programmes gouvernementaux quant à l'incorporation segmentée. Nous affirmons leur influence très grande sur la reformulation identitaire des aînés d'origine libanaise.

Dans leurs rapports de recherche sur l'incorporation citoyenne des québécois d'origine arabe, Oueslati, Labelle et Antonius (2006) remarquent que "le choix du Canada comme option identitaire devient donc une posture défensive contre les Québécois issus de la majorité francophone à cause de rapports de pouvoir qui leur sont défavorables et du rejet ressenti » (p. 151). Dans le cadre de notre recherche auprès des aînés d'origine libanaise, nous arrivons à des résultats différents. Quoique plusieurs répondants ne fassent pas de distinction claire entre le contexte canadien et celui du Québec quant à la question de la citoyenneté, nous pouvons toutefois affirmer que plusieurs personnes, arrivées dans les années 1950 et 1960, ont participé de près à la Révolution tranquille, ont frémi aux aspirations de souveraineté, ont vécu les débats acerbes sur la religion et la langue.

Rappelons ici la position de Cerclé et Sonat (2002) : le processus de comparaison sociale fournit l'argumentation. Nous affirmons l'importance et l'influence de ces éléments et processus sur la reformulation identitaire des aînés d'origine libanaise. Les répondants ont construit facilement leur réseau de contacts. Leur ascension professionnelle leur a permis de s'identifier davantage à la classe moyenne québécoise. Leur maîtrise de la langue a facilité l'acquisition des codes culturels et les protocoles d'interaction avec la population d'accueil.

\section{Le Liban : nostalgie et colère}

L'analyse de nos données indique que le trauma vécu par les personnes répondantes au Liban en lien avec les crises qui ont secoué le pays et l'image négative diffusée par les médias à l'égard de leur pays d'origine a contribué à transformer leur identité. Nous rejoignons sur ce point les travaux de Festinger (1971), Tajfel (1972), Cerclé et Sonat (2002) et De La Sablonnière (2007), qui affirment que la comparaison sociale est au centre de la construction identitaire et de sa transformation et que sous l'influence de plusieurs facteurs notamment le lieu, la langue, le système des valeurs et le temps, les stratégies identitaires individuelles peuvent être privilégiées par rapport à celles du groupe d'appartenance. Ainsi, les personnes aînées interviewées abordent cette question d'identification en faisant des comparaisons diverses (sociales et temporelles) entre leur situation ici et celle qui prévalait dans le pays d'origine.

Les entrevues ont révélé une certaine opposition de la part de quelques personnes répondantes à la culture d'ici. L'héritage de la culture d'origine les accompagne quotidiennement et conditionne leurs perceptions, leurs 
jugements et leurs interactions. La compréhension et l'apprentissage de nouvelles valeurs sont parfois difficiles pour certaines, et l'adoption d'autres codes est exigeante. Pour ces raisons, l'attachement aux valeurs d'origine relève davantage, à notre avis, d’une quête nostalgique. La libanité exprimée par les répondants est composée d'émotions, de nostalgie, d'attendrissement et de regret pour le pays d'origine. Par contre, quand la méthode comparative se met en branle, la colère gronde et la critique se fait virulente sur la violence et la guerre, sur l'organisation de la vie, l'État, l'absence de politique sociale universelle, la désorganisation et le manque de structuration au Liban : peu de sensibilité sociale pour les aînés, pas de services ou de soutien au quotidien.

Il n'y a donc pas de négation radicale de la société d'origine. Par contre, il y a adoption d'autres codes par opposition et distanciation à l'égard de la désorganisation du pays d'origine.

\section{Famille et religion : de socs centraux apaisés}

La famille a toujours été un thème important dans la société libanaise. Plusieurs auteurs, comme Abou (2002), Joseph (1998) et Le Gall (2001), en témoignent. Nos résultats sur l'importance de la famille concordent avec les études de Joseph (1998) portant sur la famille libanaise. La famille est, comme nous l'avons vu, au cœur du projet migratoire. II y a une proximité affective et physique dans beaucoup de situations, mais pas trop de promiscuité, le moins possible de subordination, de dépendance servile, de sentiment d'humiliation. Même si les répondants demeurent de fervents pratiquants et que leur raison principale pour avoir immigré repose sur la réunification familiale dans un pays sûr, on peut noter pour l'identité familiale ainsi que pour le rapport à la confession une forme de "déprise ", un nouveau rapport plus serein, plus détaché. Les aînés interviewés ne tarissent pas d’éloges devant la fierté et le réconfort de voir leurs enfants travailler et vivre décemment, devant l'opportunité de voir grandir leurs petits-enfants. Ce bonheur comble les espoirs si ardemment entretenus alors qu'il a fallu fuir la guerre, l'insécurité économique et la peur de perdre les êtres chers. L'accent se concentre sur les enfants et les petits-enfants dans un nouvel espace permettant de vivre aussi pour soi, avec son conjoint, avec des amis, un espace donnant accès à des projets personnels, à une liberté nouvelle.

\section{Un processus de subjectivation très prégnant}

Un des éléments importants à signaler lors de nos entrevues fut que de nombreuses femmes aînées libanaises affirmaient vivre la maternité comme première identité. Au-dessus des ancrages confessionnels, familiaux, communautaires, de classe sociale ou autres, le fait d'être mère, principale référence identitaire, primait au Liban. Cela pouvait être une réduction essentialiste compréhensible dans une société traditionnelle bien encadrée. Ce pouvait être aussi une affirmation de l'importance de la vie, du don et de la responsabilité.

Mais encore plus étonnante fut l'affirmation par des aînés - hommes et femmes, mais d'une façon plus insistante de la part des femmes - d'un processus de subjectivation ou d'individuation provenant de la vie dans la société d'accueil. Comme le souligne Touraine, entre autres dans Pourrons-nous vivre ensemble égaux et différents? (Touraine, 1997), ce processus se fait par une critique des contraintes, par une critique de la domination, en développant l'amour de soi, la reconnaissance comme étant sujet-acteur en combinant les ancrages culturels, la rationalité pour vivre ensemble, en toute liberté. Ce processus est un travail sur soi permettant à quelques-uns de devenir sujets-acteurs de leur vie et des orientations de ce qui vient. Ce travail exige de se distancier des codes et valeurs dominants ou imposés, de développer une estime et une confiance en soi, et de reconnaître les autres comme travaillant aussi à devenir sujets affranchis. Les façons d'exprimer ce processus se réfèrent à des espaces de liberté et d'autonomie qui sont créés, à une façon de se "déprendre » de la dépendance à l'égard des appartenances communautaristes étouffantes et conflictuelles en permanence, du mari, des enfants et enfin à un sentiment de liberté et de renouveau loin des conventions, surtout pour les femmes, mais aussi pour les hommes. Dans le cas des femmes, ce qui est frappant, c'est le pouvoir de devenir sujet-actrice de leur propre vie.

\section{Conclusion}

À travers cette recherche, nous avons réussi à saisir la dynamique entre l'expérience de la vie au Liban et celle dans la société d'accueil. Notre surprise fut grande en découvrant que, contrairement aux différents discours des théoriciens, politiciens, historiens et sociologues, deux options identitaires - la première en lien avec la religion, la 
seconde est en lien avec l'identité arabe - semblent être faibles, même absentes. Nous avons été étonné de savoir que la place de la confession s'est amenuisée, alors que les rapports familiaux ont été mis de l'avant dans le quotidien des personnes âgées. Sur le plan de l'affirmation identitaire arabe, le contexte géopolitique au Liban a influencé la construction d'un discours autour de l'Oumma (la mère patrie), affirmant d'un coup l'émergence d'un nationalisme arabe fort. Fait remarquable, le pôle identitaire arabe n'est pas ressorti d'une façon convaincante, nonobstant la diversité d'appartenance (religion, sexe, classe sociale) des personnes interrogées et de leur origine commune, où l'arabité serait un socle de l'identité, comme nous l'avons démontré plus haut à travers les propos de Sélim Abou.

La dynamique de rupture dans le discours des personnes âgées alimente positivement leur insertion et acceptation de la société d'accueil. Ces expériences sont venues remettre en question leur identité dans un moment où elles ont eu le sentiment d'être mises à l'écart dans leur pays d'origine, comme certaines l'ont mentionné. Elles ont trouvé les valeurs qu'elles recherchaient, en même temps que leurs habitudes de vie familiale, sociale et culturelle libanaise. Si l'on interrogeait des personnes âgées dans d'autres contextes, par exemple celles prises en charge dans les réseaux privés et publics de résidences pour personnes âgées, arriverions-nous à des résultats semblables?

Plusieurs questions restent en suspens. Les répondants semblent avoir un attachement très significatif envers les valeurs familiales. Quelles configurations prennent les pratiques des jeunes de la deuxième génération à cet égard? Sachant que les personnes âgées arrivées dans les années 1950 et 1960 étaient actives dans société québécoise, qu'en est-il aujourd'hui de leurs enfants et petits-enfants?

\section{Bibliographie}

Abou, S. (2002). L'identité culturelle suivie de Cultures et Droits de l'Homme. Beyrouth : Université Saint-Joseph.

Ammoune, D. (2004). Histoire du Liban contemporain. Paris : Fayard, 264 p.

Berger, P. et Luckmann, T. (1986). La construction sociale de la réalité. Paris : Méridiens Klienksieck.

Berry, J. (2000). Acculturation et identité. Dans Pluralité des cultures et dynamiques identitaires (p. 129-148). Paris : L'Harmattan.

Camilleri, C. et al. (1990). Stratégies identitaires. Paris : Presses universitaires de France.

Cerclé, A. et Sonat, A. (2002). Psychologie sociale. Paris : Dunod.

Charpentier, M. et Quéniart, A. (2011). Repenser la vieillesse, renouveler les pratiques. Nouvelles pratiques sociales, 24(1), 15-20.

De la Sablonnière, R. et al. (2007). Vers une intégration réciproque des identités culturelles : un sens à la crise symptomatique des accommodements raisonnables. Mémoire présenté à la Commission de consultation sur les pratiques d'accommodement reliées aux différences culturelles. En ligne à :

http://www.mapageweb.umontreal.ca/delasabr/publications/de\%20la\%20Sablonni\%C3\%A8re\%20et\%20al., \%20\%2029\%20nov\%202007.pdf

Gauthier, B. (1996). Recherche sociale, de la problématique à la collecte de données. Québec : Presses de I'Université du Québec.

Gouvernement du Québec (2015). Recueil de statistiques sur l'immigration et la diversité au Québec. Montréal : Ministère de l'immigration de la diversité et de l'inclusion.

Festinger, L. (1971). Théorie des processus de comparaison sociale. Dans C. Faucheux et S. Moskovici (dir.), Psychologie sociale : Théorique et expérimentale (p. 64-85). Paris : Mouton.

Joseph, S. (1998). Stradding Visible and Invisible Lebanese Economies. Dans R. Lobban Jr (dir.), Middle Eastern Women and Invisible Enonomy (p. 182-213). Gainesville : University Press of Florida. 
Labelle, M., Salée, D. et Frenette, G. (2001). Incorporation citoyenne et/ou exclusion? La deuxième génération issue de l'immigration haïtienne et jamaïcaine (Rapport de recherche). Toronto : Fondation canadienne des relations raciales.

Le Gall, J. (2001). La participation des femmes au processus de migration transcontinentale familiale. Le cas des Shi'ites libanais de Montréal (Thèse de doctorat inédite). Université de Montréal.

Lévesque, M. et White D. (1999). Le concept de capital social et ses usages. Lien social et politique, 41, 23-33.

Manço, A. (1999). Intégration et identité. Stratégies et positions des jeunes issus de l'immigration. Bruxelles : De Boeck.

Martiniello, M. (1995). L'ethnicité dans les sciences sociales contemporaines. Paris : Presses universitaires de France.

Oueslati, B., Labelle, M., Antonius, R. (2006). Incorporation citoyenne de jeunes Québécois d'origine arabe: conceptions, pratiques et défis. Rapport de recherche. Montréal : Université du Québec à Montréal. (Les Cahiers du Centre de recherche sur l'immigration, l'ethnicité et la citoyenneté)

Pinta, P. (1995). Le Liban. Paris : Méridien.

Portes, A. et Zhou, M. (1993). The new second generation, segmented assimilation and its variants. Annals of the American of Political and Social Sciences, 530, 74-96.

Tajfel, H. (1972). La catégorisation sociale. Dans S. Moscovici (dir.), Introduction à la psychologie sociale (p. 273302). Paris : Larousse.

Touraine, A. (1997). Pourrons-nous vivre ensemble égaux et différents? Paris : Fayard.

Waldinger, R. (1996). Still the Promised City? African-American and new immigrants in postindustrial New York. Cambridge, MA : Harvard University Press.

Waters, M. (1996). Ethnic and racial identities of second generation black immigrants in New York City. International Migration Review, 28(4), 795-820. 